\title{
THE GEOMETRIC REALIZATION OF A KAN FIBRATION IS A SERRE FIBRATION
}

\author{
DANIEL G. QUILLEN ${ }^{1}$
}

The object of this note is to prove the statement in the title which is asserted without proof in [1, Lemma 2.1].

We follow the terminology of $[2$, II, 3] except that a map of simplicial sets which is both a (Kan) fibration and a weak equivalence will be called an acyclic fibration instead of trivial fibration. The term trivial will be used as in [4] for a map which is isomorphic to the projection of a product onto one of its factors.

Lemma. Any fibration $f: X \rightarrow Y$ of simplicial sets may be factored $f=p g$ where $p$ is a minimal fibration and $g$ is an acyclic fibration.

Proof. By the theory of minimal fibrations [3] (see also [4, VI, 5.2]) there is a simplicial subset $Z$ of $X$ such that the restriction $p$ of $f$ to $Z$ is a minimal fibration and such that $Z$ is a strong deformation retract of $X$ relative to $Y$. Let $j$ be the inclusion of $Z$ in $X$ and let $g: X \rightarrow Z$ be the retraction of $X$ onto $Z$. We claim that $g$ is an acyclic fibration. Suppose given $u: \Delta^{\cdot}[n] \rightarrow X$ and $v: \Delta[n] \rightarrow Z$ with $g u=v i$, where $i$ is the inclusion $\Delta^{\cdot}[n] \rightarrow \Delta[n]$. Recall that the maps in a category form another category with commutative squares for morphisms, and let $A$ (resp. $B$ ) be the map from $i$ to $f$ given by the pair $u$, $p v$ (resp. $j g u, p v$ ). The homotopy of deformation from $i d_{x}$ to $j g$ gives a homotopy from $A$ to $B$. But the map $B$ has a lifting, namely $j v$, and so by the covering homotopy extension theorem $A$ has a lifting $r: \Delta[n] \rightarrow X$. If $i_{n}$ is the canonical $n$ simplex of $\Delta[n]$, then $g r\left(i_{n}\right)$ and $v\left(i_{n}\right)$ are two simplices of $Z$ which are homotopic relative to their common boundary and to $p$, so they coincide by the minimality of $p$. Thus $g r=v$ and $r i=u$ and we find that $g$ is an acyclic fibration.

Q.E.D.

The geometric realization of a minimal fibration is a Serre fibration because it is locally trivial $[4, \mathrm{VI}, 5.4]$ and because the geometric realization of a locally trivial map is a Serre fibration [4, VII, 1.4]. As the composition of Serre fibrations is a Serre fibration we are therefore reduced to the case where $f$ is an acyclic fibration.

In this case choose an injective map $k: X \rightarrow W$, where $W$ is a contractible Kan complex. This may be done by factoring the map

Received by the editors August 30, 1967.

1 This research was supported in part by NSF grant GP6166. 
$X \rightarrow \Delta[0]$ into a cofibration followed by an acyclic fibration. Then $(k, f): X \rightarrow W \times Y$ is injective and $\mathrm{pr}_{2}: W \times Y \rightarrow Y$ is an acyclic fibration. As $f=\operatorname{pr}_{2} \circ(k, f)$ is an acyclic fibration, $(k, f)$ has the left lifting property with respect to $f$, so $f$ is a retract of $\mathrm{pr}_{2}$. But $\left|\mathrm{pr}_{2}\right|$ is a Serre fibration because $\mathrm{pr}_{2}$ is trivial, so $|f|$ is a retract of a Serre fibration and is therefore a Serre fibration.

Q.E.D.

REMARK. The above argument may be modified to show that the geometric realization of a Kan fibration of countable simplicial sets is a Hurewicz fibration. Indeed one again reduces to proving that if $f: X \rightarrow Y$ is a locally trivial map of countable simplicial sets with fiber $F$, then $|f|$ is a Hurewicz fibration. However the proof of [4, VI, 5.4] show's that $|Y|$ is covered by open subsets $U$ which are countable $\mathrm{CW}$ complexes such that $|f|^{-1} U$ is isomorphic to the product $U \times|F|$ in the category of Kelley spaces. As $U$ and $|F|$ are countable CW complexes, the Kelley product coincides with the ordinary product (use Lemma 2.1 of [5]), so $|f|$ is a locally trivial map of spaces. Thus $|f|$ is locally a Hurewicz fibration and since a CW complex is paracompact [6], $|f|$ is a Hurewicz fibration [7].

We do not know if the countability assumptions are necessary.

\section{REFERENCES}

1. N. H. Kuiper and R. K. Lashof, Microbundles and bundles. II. Semisimplicial theory, Invent. Math. 1 (1966), 243-259.

2. D. G. Quillen, Homotopical algebra, Lecture Notes in Math. No.43, Springer, Berlin, 1967.

3. M. G. Barratt, V. K. A. M. Guggenheim and J. C. Moore. On semi-simplicial fibre bundles, Amer. J. Math. 81 (1959), 639-657.

4. P. Gabriel and M. Zisman, Calculus of fractions and homotopy theory, Springer, Verlag, Berlin 1966.

5. J. Milnor, Construction of universal bundles. I, Ann. of Math. (2) 63 (1956), 272284.

6. H. Miyazaki, The paracompactness of CW-complexes, Tohoku Math. J. (2) 4 (1952), 309-313.

7. W. Hurewicz, On the concept of fiber space, Proc. Nat. Acad. Sci. U.S.A. 41 (1955), 956-961.

Massachusetts Institute of Technology 